\title{
Effects of Locally Administered Tiludronic Acid on Experimental Periodontitis in Rats
}

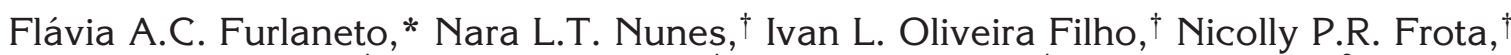
Kely O. Yamamoto, ${ }^{\dagger}$ Mario R.P. Lisboa, ${ }^{\dagger}$ Edilson Ervolino, ${ }^{\ddagger}$ Mario Taba Jr., $\S$

Rodrigo Otávio Rêgo, ${ }^{\dagger}$ and Michel R. Messora§

Background: It appears there are no studies evaluating the influence of the bisphosphonate tiludronic acid (TIL) on periodontitis. The purpose of this study is to evaluate via microtomographic, histopathologic, histometric, and immunohistochemical analyses the effects of local administration of TIL on ligature-induced periodontitis in rats.

Methods: Forty-eight rats were divided into six groups: C (control), EP (experimental periodontitis), EP-Saline, EPTIL0.1, EP-TIL0.3, and EP-TIL1. In EP, a ligature was placed around maxillary second molars. In EP-TIL0.1, EP-TIL0.3, and EP-TIL1, TIL solutions of $0.1,0.3$, and $1 \mathrm{mg} / \mathrm{kg}$ body weight, respectively, were injected into the subperiosteal palatal area adjacent to maxillary second molars every other day. EP-Saline received $0.9 \% \mathrm{NaCl}$ solution instead. Animals were euthanized at day 11 . Bone changes were evaluated by microtomographic and histometric analyses. Histopathologic analysis and immunohistochemical detection of tartrate-resistant acid phosphatase (TRAP) were also performed. Data were statistically analyzed (analysis of variance or Kruskal-Wallis, $P<0.05$ ).

Results: Histometric and microtomographic analyses (at buccal, interproximal, and furcation sites) demonstrated that EP-TIL1 presented less alveolar bone loss (ABL) than EP $(P<0.05)$, whereas EP-TIL0.1 and EP-TIL0.3 did not demonstrate significant differences in alveolar bone level compared to EP $(P>0.05)$. Also, EP-TIL1 showed significantly fewer TRAP-positive multinucleated osteoclasts than EP and EP-Saline $(P<0.05)$.

Conclusion: It can be concluded that locally administered TIL solution ( $1 \mathrm{mg} / \mathrm{kg}$ body weight) reduced alveolar bone loss in experimental periodontitis and the dosage of TIL may influence its anti-inflammatory and antiresorptive properties. J Periodontol 2014;85:1291-1301.

\section{KEY WORDS}

Administration, buccal; bone resorption; diphosphonates; osteoclasts; periodontal diseases; tiludronic acid.

\footnotetext{
* Department of Surgery and Integrated Clinic, Division of Periodontics, Dental School of Araçatuba, UNESP, São Paulo State University, Araçatuba, SP, Brazil.

$\dagger$ Federal University of Ceará, Fortaleza, CE, Brazil.

‡ Department of Basic Sciences, Division of Histology, Dental School of Araçatuba, UNESP, São Paulo State University.

$\S$ Department of Surgery and Bucco-Maxillofacial Traumatology and Periodontology, Ribeirão Preto School of Dentistry, University of São Paulo, Ribeirão Preto, SP, Brazil.
}

The presence of a periodontopathogenic dental biofilm can induce an inflammatory reaction in periodontal tissues, leading to the secretion of proinflammatory cytokines (such as interleukin [IL]-1 $\beta$ and tumor necrosis factor- $\alpha[$ TNF- $\alpha])$, prostaglandins, and matrix metalloproteinases (MMPs) by immune cells (leukocytes and macrophages) and gingival fibroblasts. ${ }^{1,2}$ These inflammatory mediators stimulate the alveolar bone resorption performed by osteoclasts and also the apical migration of the junctional epithelium, characterizing periodontitis. The severity and progression of this disease are modified in genetically susceptible individuals and in the presence of immunoderegulating risk factors such as diabetes mellitus and smoking. ${ }^{3}$ For these susceptible patients, most of the periodontal breakdown may occur because of the inflammatory response of the host. ${ }^{4}$

The prerequisites for successful conventional periodontal treatment are the patient's cooperation, adequate oral hygiene, ${ }^{5}$ and the mechanical debridement of tooth surfaces. ${ }^{6,7}$ Recently, a new approach for periodontal treatment, comprising the control of the host response to bacterial aggression, has been studied. ${ }^{4}$ Bisphosphonates (BPs) are synthetic chemical drugs very efficient in the treatment of some bone

doi: $10.1902 / j o p .2014 .130581$ 
diseases such as osteoporosis, Paget's disease, multiple myeloma, hypercalcemia of malignancy, and bone metastasis, decreasing the risk of fractures. ${ }^{8-10}$ The proven efficacy of BPs to inhibit osteoclastic bone resorption ${ }^{11}$ has led to their use in the management of periodontitis as a host-modulating factor in the perspective of reducing alveolar bone loss (ABL). ${ }^{12,13}$

Tiludronic acid (chloro-4-phenyl-thiomethylene1,1-bisphosphonate) (TIL), a BP from the first generation, was characterized by dose-dependently inhibiting bone resorption in several in vivo preclinical studies, ${ }^{14,15}$ including models of thyroparathyroidectomized, ${ }^{14}$ neurectomized, ${ }^{15}$ and ovariectomized ${ }^{14}$ rats. Osteoclasts internalize this type of BP and convert them to a non-functional molecule that competes against adenosine triphosphate in cell metabolism. The intracellular accumulation of these metabolites has a cytotoxic effect and leads to osteoclast apoptosis. ${ }^{16}$ In vitro studies demonstrated that this BP also presents anti-inflammatory actions, as it can dosedependently inhibit IL- 6 synthesis by osteoblasts ${ }^{17}$ and the secretion of IL-1 $\beta$, IL- 6 , nitric oxide (NO), and TNF $-\alpha$ by activated macrophages. ${ }^{18}$ The inhibitory effects of TIL on important enzymes for the degradation of extracellular matrix components in periodontitis (MMP-1 and -3) were also shown in human periodontal ligament cells. ${ }^{19}$

TIL is a non-nitrogen-containing BP, which presents a low potency when compared to nitrogencontaining BPs. ${ }^{16}$ Especially for this reason, TIL is well tolerated and seems to exhibit only mild adverse effects, different from those usually associated with nitrogen-containing BPs, ${ }^{20}$ such as gastrointestinal disorders, ${ }^{21}$ ocular lesions, ${ }^{22}$ acute-phase reactions (flu-like symptoms and increase in the circulating levels of IL-1, IL-6, and TNF- $\alpha$ ), ${ }^{22}$ and osteonecrosis of the jaw. ${ }^{11,23,24}$ TIL is a safe compound with an appreciable therapeutic margin. ${ }^{25,26}$

Because periodontitis is primarily caused by local infectious factors, the drug of choice must be administered in higher doses to maintain an effective concentration at periodontal tissues. ${ }^{27,28}$ To achieve this concentration and avoid the adverse effects associated with systemic administration of BPs, some authors have defended their local administration in periodontal ${ }^{27-29}$ and peri-implant tissues. ${ }^{30}$

Because of its antiresorptive, anti-inflammatory properties and safety, TIL seems to be an efficient BP for periodontitis management. To the best of the authors' knowledge, there are no studies evaluating the influence of TIL on periodontitis. The purpose of this study is to analyze the effects of local administration of TIL on inflammatory response and ABL in rats with experimental periodontitis (EP).

\section{MATERIALS AND METHODS}

\section{Sample Size}

This study was conducted in compliance with the ethical principles of animal experimentation, as well as standards for the didactic-scientific practice of vivisection and the Universal Declaration of Animal Rights by the United Nations Educational, Scientific, and Cultural Organization. The present study was conducted after review and approval by the Ethics Committee on Animal Research at Federal University of Ceará-UFC, Fortaleza, CE, Brazil (protocol 028/2011).

A power calculation was performed to determine the sample size. The animal was considered the study unit. The sample size was determined to provide $80 \%$ power to recognize a significant difference of $20 \%$ among groups and the standard deviation of $15 \%$ with a 95\% confidence interval ( $\alpha=0.05)$, considering the change in alveolar bone level as the primary outcome variable. Therefore, a sample size of eight animals per group was required.

\section{Experimental Model}

Forty-eight 3- to 4-month-old male Wistar rats (Rattus norvegicus, albinus) weighing 250 to $300 \mathrm{~g}$ were used (Central Animal Facility of the Federal University of Ceará-(IFC) in this study. The rats were kept in a room with a 12-hour light/dark cycle with temperatures between $22^{\circ} \mathrm{C}$ and $24^{\circ} \mathrm{C}$. Throughout the experiment, the animals were housed in plastic cages and fed with selected solid diet and water ad libitum. They were randomly assigned to one of six experimental groups $(n=8)$, according to the following protocol: 1) C (control): periodontitis was not induced and there was no administration of TIL; 2) EP (experimental periodontitis): EP was induced with a ligature and there was no administration of TIL; 3) EP-Saline: EP was induced with a ligature and saline solution ( $\mathrm{NaCl} 0.9 \%$ ) was locally administered; 4 ) EP-TIL0.1: EP was induced with a ligature and TIL solution at a dosage of $0.1 \mathrm{mg} / \mathrm{kg}$ body weight was locally administered; 5) EP-TIL0.3: EP was induced with a ligature and TIL solution at a dosage of $0.3 \mathrm{mg} /$ kg was locally administered; and 6) EP-TIL1: EP was induced with a ligature and TIL solution at a dosage of $1 \mathrm{mg} / \mathrm{kg}$ was locally administered.

\section{Induction of Periodontitis With Ligature}

On day 1 , all animals were anesthetized by an intramuscular injection of xylazine $(6 \mathrm{mg} / \mathrm{kg}$ body weight) and ketamine ( $70 \mathrm{mg} / \mathrm{kg}$ body weight). After general anesthesia, each animal was placed on the operating table, which allowed keeping the rat's mouth open, facilitating access to posterior teeth of the maxillae. Except the animals from group C,

\| Rompum, Bayer Saúde Animal, São Paulo, SP, Brazil.

II Dopalen, Agribands, Paulínia, SP, Brazil. 
a model for EP described previously ${ }^{31,32}$ was performed. A nylon ligature ${ }^{\#}$ was placed around the cervical area of the left maxillary second molar of each rat, remaining supragingivally at both buccal and lingual faces. The knot was positioned at the buccal face of the tooth. In EP-TIL0.1, EP-TIL0.3, and EP-TIL1 groups, $20-\mu \mathrm{L}$ TIL* * solutions $(0.1,0.3$, and $1 \mathrm{mg} / \mathrm{kg}$ body weight, respectively) were injected into the subperiosteal palatal area adjacent to the left maxillary second molars on days 1, 3, 5, 7, and 9 . Animals in the EP-Saline group received $20 \mu \mathrm{L}$ of $0.9 \% \mathrm{NaCl}$ solution instead. Throughout the experimental period, the animals were weighed every other day, and the doses of TIL were adapted accordingly.

The animals were euthanized under anesthesia with a final solution of xylazine $(30 \mathrm{mg} / \mathrm{kg}$ body weight) and ketamine (240 mg/kg body weight) at day 11 . The left hemimaxillae were excised, fixed in $4 \%$ formaldehyde for 24 hours, and rinsed with water.

\section{Micro-CT Analyses}

Non-demineralized specimens were scanned by a cone-beam microcomputed tomography (micro-CT) system. ${ }^{\dagger \dagger}$ The $\mathrm{x}$-ray generator was operated at an accelerated potential of $60 \mathrm{kV}$ with a beam current of $165 \mu \mathrm{A}$ and an exposure time of $630 \mathrm{~ms}$ per projection. Images were produced with a voxel size of $6 \times 6 \times 6 \mu \mathrm{m}$.

Using appropriate software, ${ }^{\neq} \neq$the generated threedimensional models were rotated into a standard position according to the following criteria: 1 ) in the transaxial plane, the maxillary second molar (M2) had its axis vertically positioned; 2 ) in the sagittal plane, the occlusal surface of M2 was horizontally positioned; and 3) in the coronal plane, alveolar bone was vertically oriented, with the roots of $M 2$ pointing up. Linear measurements on alveolar bone levels were performed at four different sites: buccal, palatal, furcation, and interproximal. For buccal and palatal sites, on the transaxial image passing through the mesial root of $\mathrm{M} 2$, the linear distances from the cemento-enamel junction (CEJ) to the buccal/palatal alveolar bone crest $(A B C)$ were measured (Fig. 1A). For the furcation site, on the sagittal image passing through both mesial and distal roots of $M 2$, ABL was assessed by measuring the distance between the roof of the furcation and the $A B C$ in the furcation area (Fig. 1B). For the interproximal site, the coronal dataset was analyzed using software. $\S \S$ The distance between the last image showing the $A B C$, between the maxillary first molar and $\mathrm{M} 2$, and the first image showing the CEJ of M2, was measured (Figs. 1C and 1D). Linear measurements were performed by one calibrated examiner (ML) who was masked to the experimental groups and treatments rendered.

\section{Histopathologic and Histometric Analyses of Periodontal Tissues}

The specimens were decalcified in 10\% EDTA solution. After complete decalcification, the specimens were processed and embedded in paraffin. Serial sections, $5 \mu \mathrm{m}$ thick, were obtained in a mesio-distal direction. The sections were stained with hematoxylin and eosin (H\&E) for analysis by light microscopy.

The histopathologic analysis was performed by a certified histologist (EE), who evaluated the following parameters: 1) nature and degree of inflammation of periodontal tissues; 2) influence of the inflammatory process on surrounding tissues; 3) presence and extension of tissue necrosis; 4) presence and extension of osseous sequestrum; 5) presence and extension of root resorption; 6) vascular status; and 7) cellularity pattern of epithelial, connective, bone, and hematopoietic tissues.

For histometric analysis, sections representing the most central buccal-lingual portion in the area between first and second left maxillary molars were selected. The images of the histologic sections were captured by a digital camerall| connected to a light microscope III $_{\text {with }}$ a magnification $\times 40$. The digital images were saved on a computer and then analyzed using appropriate software. ${ }^{\#}$ To assess the interproximal alveolar bone level, a line connecting the CEJ of the first molar to the CEJ of the second molar was drawn. Then a second line, perpendicular to the first one, was drawn connecting the first line to the coronal portion of the interdental bone crest. The histometric analysis was performed by one calibrated examiner (NN) who was masked to the experimental groups and treatments rendered.

\section{Immunohistochemical Analyses for Detection of TRAP}

The histologic sections were deparaffinized and rehydrated through a graded series of ethanol. For antigen retrieval, the slides were incubated in a citrate buffer solution $(10 \mathrm{mM}, \mathrm{pH} 6.0)$ in a pressurized chamber*** at $95^{\circ} \mathrm{C}$ for 20 minutes. At the end of each stage of the immunohistochemical reaction, the histologic slides were washed with phosphatebuffered saline $(0.1 \mathrm{M}, \mathrm{pH} 7.4)$. Subsequently, the slides were immersed in 3\% hydrogen peroxide for 1 hour and then $1 \%$ bovine serum albumin for 12 hours to block the endogenous peroxidase and non-specific sites, respectively. The slides containing samples of each of the experimental groups were incubated

\footnotetext{
\# 3-0, Technofio, Goiânia, GO, Brazil.

* Tildren, Ceva Saúde Animal, Paulínia, SP, Brazil.

$\dagger$ Skyscan 1172, Bruker, Kontich, Belgium.

邦 Data Viewer, v.1.5.0, Bruker.

\& CT-Analyzer, v.1.13.5.1+, Bruker.

Ill C-SHG, Nikon Digital Sight DS-2MV, Nikon, Tokyo, Japan.

II Eclipse E200 MVR, Nikon.

\#\# ImageJ, National Institutes of Health, Washington, DC.

*** Decloaking Chamber, Biocare Medical, Concord, CA.
} 

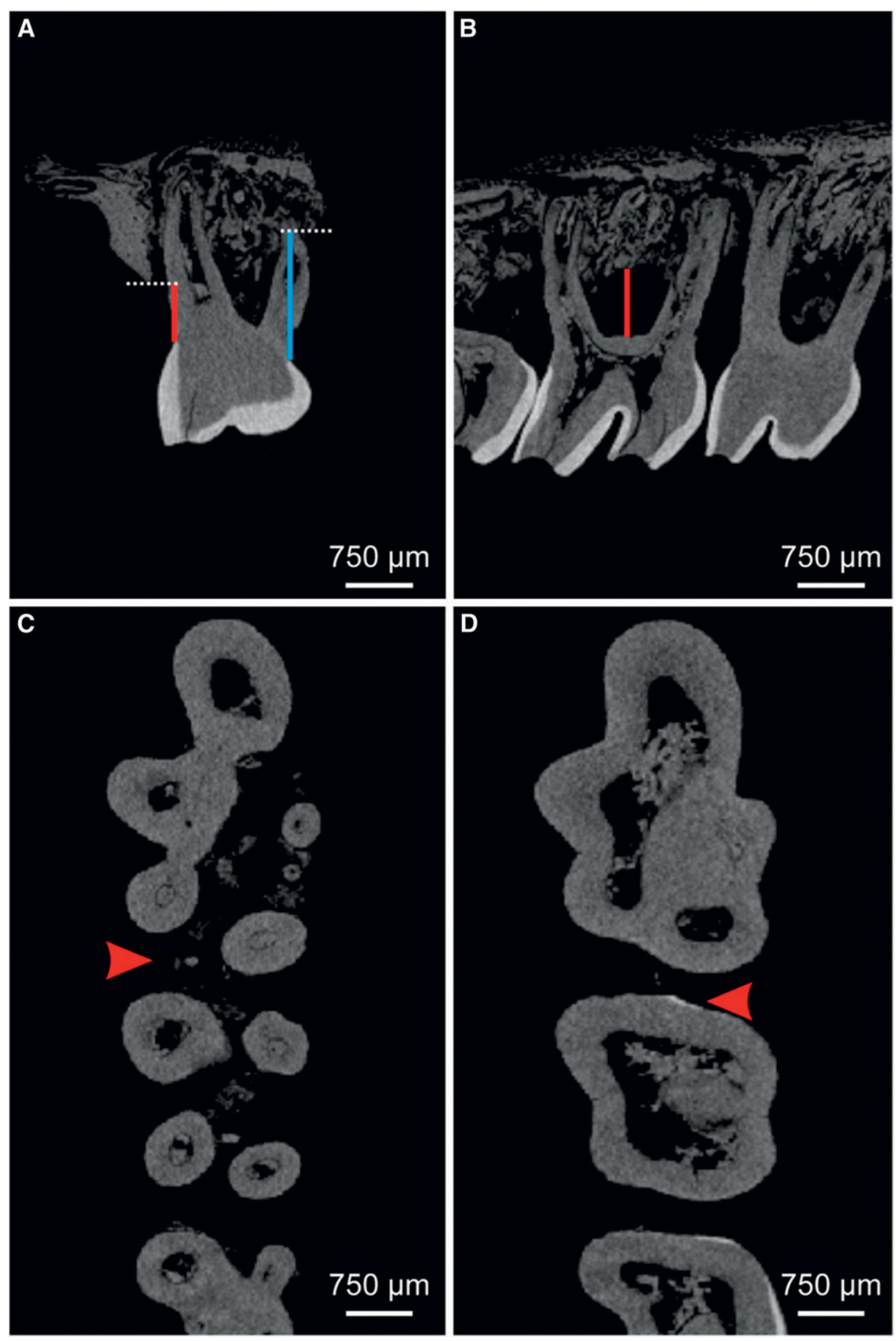

\section{Figure I.}

Micro-CT images for linear measurements in the area of the maxillary second molar. In the transaxial plane, buccal and palatal alveolar bone levels (blue and red lines, respectively) $(\boldsymbol{A})$. In the sagittal plane, alveolar bone level in furcation area (red line) (B). In the coronal dataset, the last image showing alveolar bone crest (red arrowhead) (C) and the first image showing CEJ (red arrowhead) (D). Scale bar $=750 \mu \mathrm{m}$. using chromogen 3,3'-diaminobenzidine $\S \S \S$ and counterstained with hematoxylin. All samples were accompanied by a negative control (specimens subjected to the aforementioned procedures but without the primary antibodies).

Histologic sections were evaluated under light microscopy with an optical microscope IIIII by $^{\text {by }}$ one calibrated examiner (FF) who was masked to the experimental groups and treatments rendered. The region to be analyzed included an area of $1,000 \mu \mathrm{m}^{2}$ located at the center of the interdental alveolar septum between the first and second maxillary molars. The coronal limit of this area was the $\mathrm{ABC}$, from which the region analyzed extended apically for $1,000 \mu \mathrm{m}$. The immunolabeling was defined as the brownish areas present in the cytosolic compartment of the cells. TRAPpositive cells were counted at $\times 200$ magnification and distinguished as TRAP-positive multinucleated osteoclasts (mature osteoclasts) and TRAP-positive mononuclear cells.

\section{Examiner Calibration}

To estimate the intra- and interexaminer error, the analyses were performed by two examiners (NPRF, KOY) who were masked to the experimental groups and treatments rendered. A second sample was measured again 48 hours after the first measurement. The paired $t$ test was used to calculate the intraexaminer error. A Pearson correlation analysis between the data obtained by the two examiners was also performed. $P>0.05$ in paired $t$ test and $r>0.90$ with the primary antibody goat anti-rat tartrateresistant acid phosphatase (TRAP) ${ }^{\dagger \dagger}$ for 24 hours. The sections were then incubated with a biotinylated secondary antibody for 2 hours and subsequently treated with a streptavidin-horseradish peroxidase conjugate for 1 hour. ${ }^{\ddagger \ddagger}$ The reaction was developed values in the Pearson correlation test were considered to estimate the feasibility of the proposed method.

\footnotetext{
$\dagger \dagger \dagger$ SC-30833, Santa Cruz Biotechnology, Santa Cruz, CA.

$\neq \neq \ddagger$ Universal Dako Labeled HRP Streptavidin-Biotin Kit, Dako Laboratories, Carpinteria, CA.

$\S \S \S$ DAB Chromogen Kit, Dako Laboratories.

||||| Axiostar Plus, Carl Zeiss Microlmaging, Göttingen, Germany.
} 


\section{Statistical Analyses}

The data obtained in the analyses were grouped and presented as means \pm standard deviations. The significance of differences among groups for ABL and number of TRAP-positive multinucleated osteoclasts was verified by analysis of variance (ANOVA) followed by post hoc Tukey test. The significance of differences among groups for number of TRAPpositive mononuclear cells was verified by the Kruskal-Wallis test. The significance level was set at $P=0.05$ in all tests.

\section{RESULTS}

All animals tolerated the experimental procedures well and remained healthy throughout the experimental period. No significant differences regarding body weight were observed among groups (ANOVA: $P>0.05)$.

\section{Examiner Calibration}

There were no significant differences among the measurements performed by the same examiner in all analyses (i.e., micro-CT, histometric, and immunohistochemical) when the first and second evaluations were compared $(P>0.05)$. There was also a significant correlation between the measurements obtained by the two examiners $(r>0.90)$.

\section{Micro-CT and Statistical Analyses}

Greater ABL was observed in EP compared to group C (Figs. 2A through 2D) at all sites analyzed (buccal, palatal, furcation, and interproximal; $P<0.05)$. At buccal, furcation, and interproximal sites, EP-TIL1 presented less ABL than the EP group $(P<0.05)$, whereas the other groups treated with TIL (EP-TIL0.1 and EPTIL0.3) did not demonstrate significant differences in alveolar bone level compared to EP $(P>0.05)$.

\section{Histopathologic Analyses of Periodontal Tissues}

In group $C$, junctional and sulcular epithelia remained intact. Some inflammatory cells were present in the coronal portion of the subepithelial connective tissue. The gingival connective tissue was very fibrous and full of fibroblasts. Periodontal ligament was composed of a great amount of collagen fibers, fibroblasts, and blood vessels. Sharpey's fibers were present in both cementum and alveolar bone. Cementum surface was completely intact and covered by cementoblasts.

Bone surface of the interdental septum was slightly irregular and saturated with osteoblasts or bone-lining cells. In these areas, extremely thick bone trabeculae were observed delimiting small medullar paces replete with hematopoietic bone marrow (Figs. 3A and 3B).

All groups with induction of periodontitis (EP, EP-Saline, EP-TIL0.1, EP-TIL0.3, and EPTIL1) presented loss of the interdental papillae and apical migration of the junctional epithelium (Figs. 3C through 3L). In EP and EP-Saline, gingival connective tissue presented intense inflammatory infiltrate, mainly composed of lymphocytes and plasma cells. In this area, the main groups of collagen gingival fibers were completely disorganized. Areas of active root resorption were very common, primarily in the specimens of the EP-Saline group. Narrow interdental alveolar septa were observed, with external irregular contours due to the presence of many resorption lacunae full of osteoclasts
Figure 2.

Micro-CT analysis. Means \pm standard deviations of the alveolar bone level of the specimens, with comparisons among groups, at buccal $(\boldsymbol{A})$, furcation $(\boldsymbol{B})$, interproximal $(\boldsymbol{C})$, and palatal $(\boldsymbol{D})$ sites. Same letters indicate no significant differences among groups (ANOVA: Tukey, $\mathrm{P}<0.05$ ). 


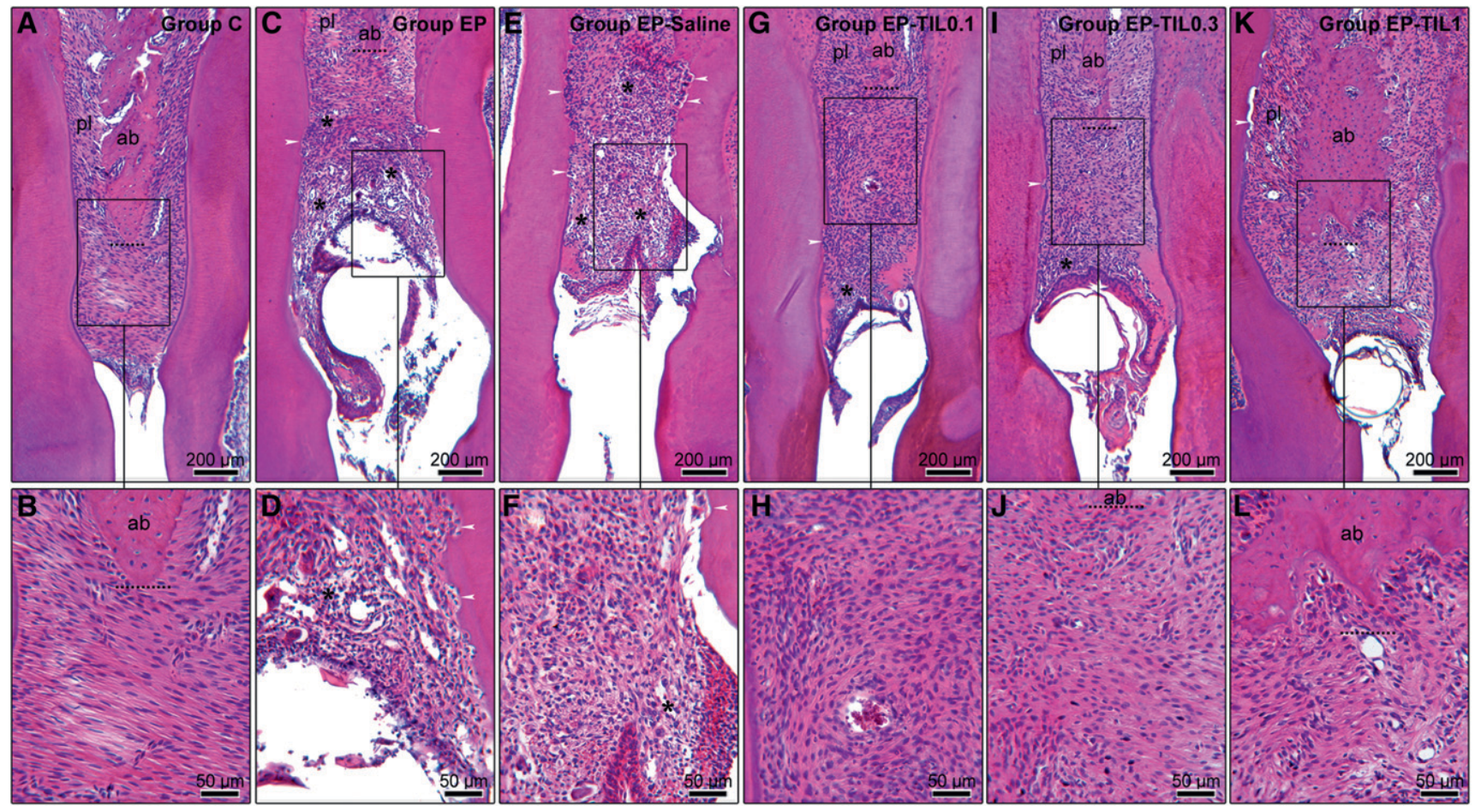

\section{Figure 3.}

Photomicrographs of periodontal tissues in interproximal areas between first and second molars: group C $(\boldsymbol{A}$ and $\mathbf{B})$; EP (C and D); EP-Saline (E and $\mathbf{F})$ EP-TILO.I (G and H); EP-TILO.3 (I and J); EP-TILI (K and $\mathbf{L}$ ). ab = alveolar bone; * = intense inflammatory infiltrate; dotted line $=A B C$ level; pl = periodontal ligament; white arrowhead = area of root resorption. Scale bars: A, C, E, G, I, and K= $200 \mu \mathrm{m} ; \mathrm{B}, \mathrm{D}, \mathrm{F}, \mathrm{H}, \mathrm{J}$, and L $=50 \mu \mathrm{m}$. (H \& E stain.)

in active resorption (Figs. 3C through 3F). In several specimens of the EP-Saline group, interdental septa were resorbed up to the level of the maxillary basal bone (Figs. 3E and 3F).

In EP-TIL0.1 and EP-TIL0.3, the magnitude of the inflammatory responses was comparatively slighter than the responses presented by the EP and EPSaline groups. Furthermore, EP-TIL0.1 and EPTIL0.3 presented inflammatory infiltrate restricted to the area adjacent to the ligature, whereas apical to this area, the connective tissue was organized with a great amount of collagen fibers, fibroblasts, blood vessels, and scarce inflammatory cells. Areas of active root resorption were also observed in some specimens of these groups. Interdental alveolar septa were covered by several osteoclasts and presented irregular outer contours (Figs. 3G through 3J). In EP-TIL1, slight inflammation was observed in the supporting tissues. Gingival connective tissue was composed of a connective tissue rich in collagen fibers, fibroblasts, and blood vessels, with mononuclear inflammatory cells especially in the area adjacent to the ligature. Areas of root resorption were not common in the specimens of this group. Interdental alveolar septa were thicker and presented a rather regular contour and small amount of osteoclasts (Figs. 3K and 3L).

\section{Histometric and Statistical Analyses}

Five specimens could not be analyzed with histometrics due to interference in laboratory processing. Therefore, the final sample of groups C, EP-Saline, EP-TIL0.1, EP-TIL0.3, and EP-TIL1 was composed of seven animals. The primary variable, alveolar bone level, achieved 0.99 power value with the present data and sample size.

Animals from EP $(1.35 \pm 0.45 \mathrm{~mm})$, EP-Saline $(1.67 \pm 0.36 \mathrm{~mm})$, and EP-TIL0.1 (1.62 $\pm 0.40 \mathrm{~mm})$ groups presented ABL significantly greater than the animals from group $C(0.64 \pm 0.28 \mathrm{~mm}, P<0.05)$. However, the animals from EP-TIL0.3 $(0.96 \pm 0.27$ $\mathrm{mm})$ and EP-TIL1 $(0.34 \pm 0.11 \mathrm{~mm})$ did not show statistically significant differences regarding $\mathrm{ABL}$ compared to group $C(P>0.05)$. The treatment with $1 \mathrm{mg} / \mathrm{kg}$ body weight (EP-TIL1) was the only treatment to provide a significant decrease in $\mathrm{ABL}$ in relation to the EP group $(P<0.05)$. Data obtained from histometric analyses and the comparisons among groups are depicted in Figure 4.

\section{TRAP and Statistical Analyses}

The immunohistochemical technique used for detecting TRAP yielded high specificity, as evidenced by the total absence of labeling in the negative controls for the immunohistochemical reactions. The immunoreactive cells presented a brownish color 


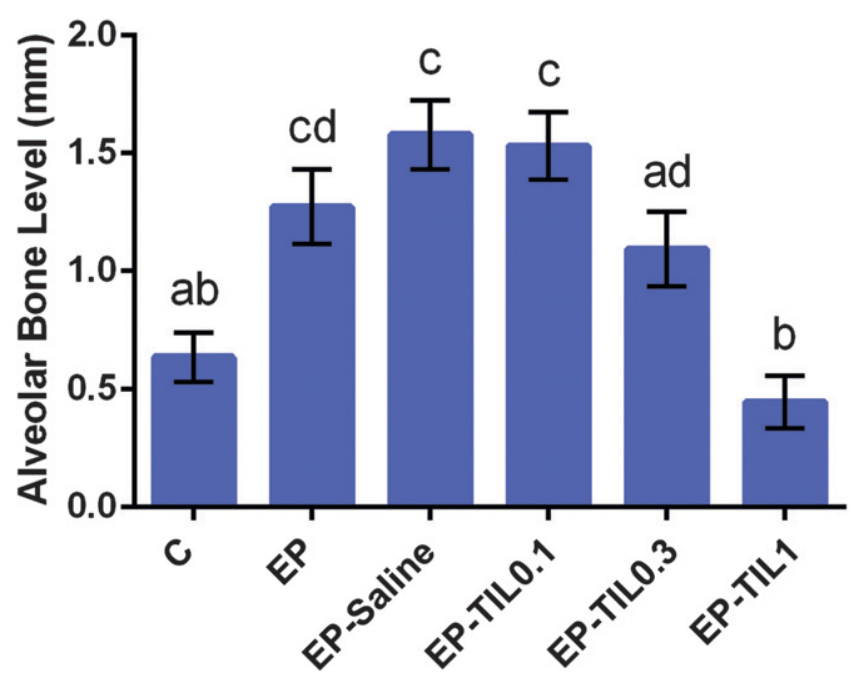

Figure 4.

Histometric analysis. Means \pm standard deviations of alveolar bone level in the interproximal areas between first and second molars, with comparisons among groups. Same letters indicate no significant differences among groups (ANOVA: Tukey, P <0.05).

that was confined to the cytosolic compartment (Figs. 5A and 5B). Immunolabeling for TRAP was present in TRAP-positive mononuclear cells (Fig. 5B) and TRAP-positive multinucleated osteoclasts (Fig. 5A).

There were no significant differences in the amount of TRAP-positive mononuclear cells among the experimental groups $(P>0.05)$. Animals from EP $\left(37.50 \pm 21.11\right.$ cells $\left./ \mathrm{mm}^{2}\right)$ and EP-Saline (54.67 \pm 27.43 cells $/ \mathrm{mm}^{2}$ ) groups presented a quantity of TRAP-positive multinucleated osteoclasts significantly greater than the animals from group C (5.00 \pm 3.34 cells $\left./ \mathrm{mm}^{2} ; P<0.05\right)$. The treatment with $1 \mathrm{mg} /$ $\mathrm{kg}$ body weight (EP-TIL1; $10.25 \pm 1.50$ cells $/ \mathrm{mm}^{2}$ ) was the only treatment to demonstrate a significant decrease in the amount of TRAP-positive multinucleated osteoclasts in relation to EP and EP-Saline groups $(P<0.05)$. Data obtained from immunohistochemical analyses for detection of TRAP and the comparisons among groups are depicted in Figures 5 and 6.

\section{DISCUSSION}

Based on current knowledge about the mechanisms of action of BPs, their use in periodontal research shows a promising method of managing periodontal diseases by modifying the host response. ${ }^{4}$ The BP tiludronic acid, which combines anti-inflammatory and antiresorptive properties, has not been studied in periodontitis until now, to our knowledge. In this context, the purpose of the present study is to evaluate the effects of local administration of TIL on ligature-induced periodontitis in rats.
The model of EP used in this study, already reported previously, ${ }^{31,32}$ allows a successful induction of the disease. In the animals of group EP (which received the ligature), ABL, periodontal attachment loss, and moderate-to-severe inflammatory infiltrate were observed. These findings were not detected in the animals from the control group, which did not receive any intervention. It is also important to emphasize that this animal model is presumed to induce maximal periodontitis; therefore, slowly progressive human cases may respond differently to the treatment evaluated in the present study.

To the best of our knowledge, only two histologic studies $^{27,29}$ demonstrated the influence of local administration of BPs on periodontitis, an approach that presents the advantage of avoiding possible systemic adverse effects of the drugs. In the present study, $\mathrm{ABL}$ and the magnitude of the inflammatory response were decreased when TIL solutions at the dosage of 1 $\mathrm{mg} / \mathrm{kg}$ body weight were locally applied. This result corroborates the findings of previous studies in which other types of BPs were locally applied. ${ }^{27,29}$ With the same technique of palatal administration used in the present study, Mitsuta et al. ${ }^{27}$ administered the BP clodronate and demonstrated its ability to prevent bone resorption and decrease the number of osteoclasts in rats with EP. Goya et al. ${ }^{29}$ applied olpadronate solutions in the depth of the gingival sulcus at the level of the furcation around rat molars with periodontitis. This treatment inhibited ABL and caused marked morphologic changes in the cytoplasm of osteoclasts, which indicated apoptosis. ${ }^{29}$ The present results also demonstrate that the local treatment influenced osteoclasts. TIL did not alter the amount of TRAP-positive mononuclear cells, which indicates that there was no interference in the recruitment of monocytes/macrophages and maturation of cells of the osteoclast lineage, as demonstrated by Murakami et al. ${ }^{33}$ The amount of TRAP-positive multinucleated osteoclasts was increased in the presence of periodontitis, resulting in greater ABL in EP and EP-Saline groups. On the other hand, treatment with $1 \mathrm{mg} / \mathrm{kg}$ body weight of TIL (EP-TIL1) was able to reduce the number of mature osteoclasts, presumably through cytotoxic effects, leading to the apoptosis of such cells, ${ }^{16,34}$ which reduced bone resorption and contributed to the preservation of alveolar bone.

Although nowadays TIL is not widely used in the treatment of generalized bone diseases, probably due to the emergence of more potent nitrogen-containing BPs (aminobisphosphonates), ${ }^{20}$ it seems to present excellent pharmacologic properties for the treatment of periodontitis. Actually, various BPs differ greatly in their pharmacologic properties and effects on inflammatory processes. ${ }^{18}$ It was reported that BPs can 


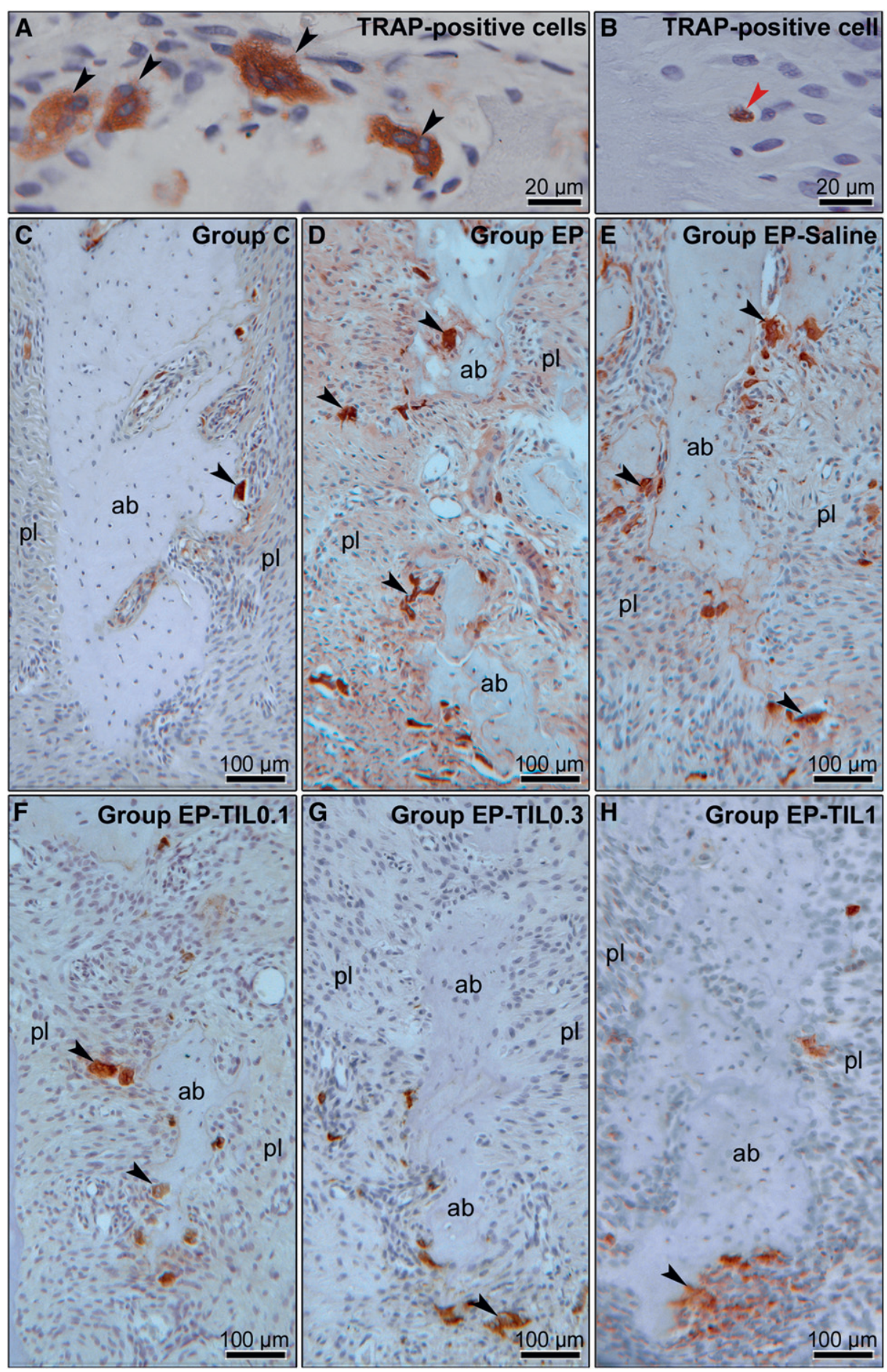

Figure 5.

TRAP immunolabeling in interproximal areas between first and second molars. Photomicrographs showing immunolabeling pattern of TRAP in TRAP-positive multinucleated osteoclasts (A) and TRAP-positive mononuclear cells (B). (C through $\boldsymbol{H}$ ) Photomicrographs showing TRAP-positive multinucleated osteoclasts in periodontal tissues in the interproximal areas between first and second molars: group $C(C)$; EP (D); EP-Saline (E); EP-TILO.I (F); EP-TILO.3 (G); EP-TILI (H). ab = alveolar bone; black arrowhead = TRAP-positive multinucleated osteoclast; $p l=$ periodontal ligament; red arrowhead = TRAP-positive mononuclear cell. Scale bars: $A$ and $B=20 \mu \mathrm{m} ; C$ through $H=100 \mu \mathrm{m}$. (Hematoxylin counterstaining.) be grouped into those that are metabolized by cells and that are capable of inhibiting cytokine and NO secretion from macrophages, thus having potential anti-inflammatory properties, and those that are aminobisphosphonates, which are not metabolized but can actually enhance the production of inflammatory cytokines following macrophage activation. ${ }^{18}$ High dosages of aminobisphosphonates stimulate local release of proinflammatory cytokines such as IL- $1 \beta$ and IL6 in periodontal tissues, which may increase periodontal inflammation and breakdown and prevent periodontal healing. ${ }^{35-37}$ It has also been suggested that nitrogen-containing classes of BPs could reduce collagen production and thus block the reconstitution of the extracellular matrix of injured periodontal tissues. ${ }^{4}$ Because TIL, a non-nitrogen-containing $\mathrm{BP}$, was used in this study, the possibility of occurrence of these undesirable effects was minimized. 24,38,39 Calcified tissues appear to be the main target for deposition of TIL. ${ }^{40}$ It is well known that IL- 6 stimulates bone resorption, ${ }^{41}$ induces osteoclast formation, ${ }^{42}$ and inhibits bone formation and that TIL is capable of inhibiting IL- 6 synthesis by osteoblasts. ${ }^{17}$ Furthermore, studies have shown that TIL is capable of increasing bone mineral density. ${ }^{43}$ Another factor that favors the choice for this BP is that TIL presents a long skeletal-retention time, leading to a marked, persistent biologic effect. ${ }^{44}$ Moreover, a serious adverse effect associated with the use of BPs, especially aminobisphosphonates, is osteonecrosis of the jaw. ${ }^{11,23,45}$ Although its pathogenesis is not completely understood yet, it is known that the greater the potency of the 


\section{A}

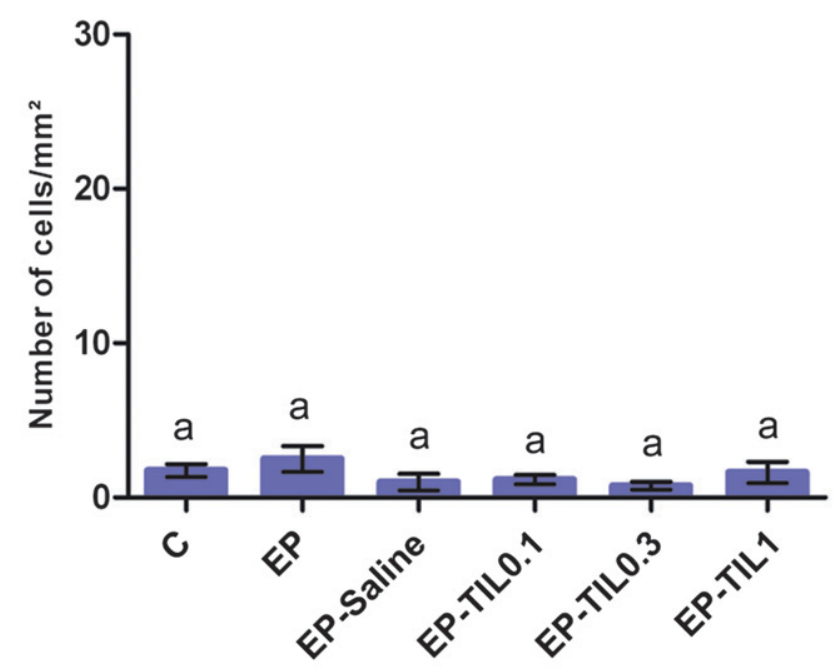

B TRAP-positive multinucleated osteoclasts

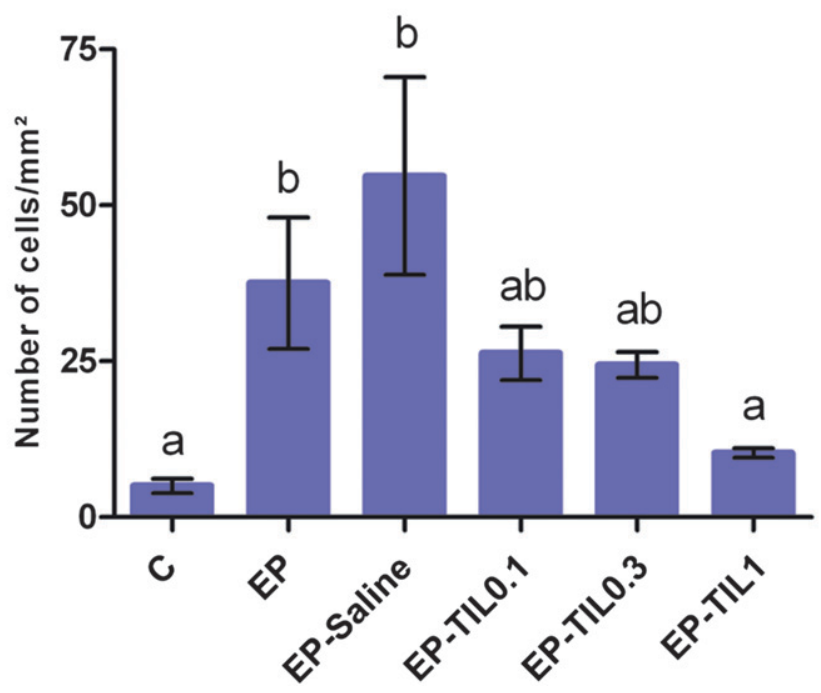

Figure 6.

Immunohistochemical analysis for detection of TRAP. Means \pm standard deviations of TRAP-positive cells, with comparisons among groups. (A) TRAP-positive mononuclear cells. (B) TRAP-positive multinucleated osteoclasts. Same letters indicate no significant differences among groups (ANOVA: Tukey, P <0.05 for TRAP-positive multinucleated osteoclasts, and Kruskal-Wallis: $\mathrm{P}<0.05$ for TRAP-positive mononuclear cells).

$\mathrm{BP}$, the greater the risk for development of osteonecrosis of the jaw. ${ }^{21}$ Therefore, TIL is a safe alternative since its potency is relatively low. ${ }^{15}$

Some studies demonstrated that the effects of BPs on decreasing cellular infiltration, number of osteoclasts, and $\mathrm{ABL}$ in periodontal tissues are dose dependent. ${ }^{36,46,47}$ Specifically in relation to TIL, in vitro and in vivo studies showed its dose-dependent effects. ${ }^{48}$ On the other hand, Mitsuta et al. ${ }^{27}$ did not find a clear dose-response relationship when clodronate was locally applied in experimental periodontitis. In the present study, histometric and micro-CT analyses demonstrate that animals treated with TIL solutions at dosages of 0.1 and $0.3 \mathrm{mg} / \mathrm{kg}$ body weight did not show alveolar bone level statistically different from animals not treated (EP group). However, animals treated with TIL solutions at the dosage of $1 \mathrm{mg} / \mathrm{kg}$ presented less ABL than the animals not treated (EP group) and were not statistically different from the animals without induction of periodontitis (group C). Thus, the local administration of TIL solutions at the dosage of $1 \mathrm{mg} / \mathrm{kg}$ body weight provided a reduction of $\mathrm{ABL}$ during the development of EP in rats, demonstrating an effect that is related to the dosage of the drug. The only exception to these results occurred when the micro-CT analysis was performed at the palatal site. It can be suggested that the choice of area to be analyzed influences the quantification of $A B L$ in the experimental model used, which demonstrates the site-specific nature of periodontitis. The micro-CT provided additional information about the effects of TIL on ABL, since a three-dimensional evaluation, including buccal and palatal sites, could be performed. The selection of the dosages used in the present study is based on previous studies with TIL in other experimental models. ${ }^{44,49}$

The findings of the present study need to be confirmed with more advanced experimental models in the phylogenetic scale and in clinical trials. As mechanical control of the dental biofilm (mainly scaling and root planing) represents the conventional periodontal treatment currently, it is necessary to investigate whether the local administration of TIL would provide additional improvements. The applicability of TIL for periodontal diseases may perhaps increase for those individuals in whom conventional periodontal therapy is not convenient, such as patients who are medically compromised, physically or mentally challenged, or elderly. ${ }^{50}$ Further studies are required also to generate dose-response curves and evaluate different therapeutic regimens, since only one regimen is analyzed in the present study (administration every other day for 11 days). Because BPs are known to bind strongly to hydroxyapatite and to remain in bone tissue for long periods of time, ${ }^{51}$ it is possible to obtain an effect of the same magnitude by administering the drugs less frequently and/or for a shorter duration. ${ }^{52}$ It is also necessary to evaluate how TIL influences periodontal tissues, investigating which inflammatory mediators are involved in the modulation of the response.

\section{CONCLUSION}

Within the limits of this study, it can be concluded that locally administered TIL solutions (1 mg/kg body weight) reduced alveolar bone loss in experimental periodontitis, and that the dosage of TIL may 
influence its anti-inflammatory and antiresorptive properties.

\section{ACKNOWLEDGMENTS}

The authors thank the National Council for Research and Technological Development (CNPq, Brasília, DF, Brazil, Process 481237/2010-0) and the Foundation for Support in Scientific and Technological Development of Ceará (FUNCAP, Fortaleza, CE, Brazil, Process 0031-00116.01.00/10) for supporting this study. The authors report no conflicts of interest related to this study.

\section{REFERENCES}

1. Socransky SS, Haffajee AD, Cugini MA, Smith C, Kent RL Jr. Microbial complexes in subgingival plaque. J Clin Periodontol 1998;25:134-144.

2. Page RC, Kornman KS. The pathogenesis of human periodontitis: An introduction. Periodontol 2000 1997; 14:9-11.

3. Kornman KS. Mapping the pathogenesis of periodontitis: A new look. J Periodontol 2008;79(Suppl. 8):1560-1568.

4. Badran Z, Kraehenmann MA, Guicheux J, Soueidan A. Bisphosphonates in periodontal treatment: A review. Oral Health Preu Dent 2009;7:3-12.

5. Axelsson P, Lindhe J. Effect of controlled oral hygiene procedures on caries and periodontal disease in adults. Results after 6 years. J Clin Periodontol 1981;8:239-248.

6 . Lindhe J, Nyman S. Scaling and granulation tissue removal in periodontal therapy. J Clin Periodontol 1985;12:374-388.

7. Kalpidis CD, Ruben MP. Treatment of intrabony periodontal defects with enamel matrix derivative: A literature review. J Periodontol 2002;73:1360-1376.

8. Reddy MS, Geurs NC, Gunsolley JC. Periodontal host modulation with antiproteinase, anti-inflammatory, and bone-sparing agents. A systematic review. Ann Periodontol 2003;8:12-37.

9. Kornman KS. Host modulation as a therapeutic strategy in the treatment of periodontal disease. Clin Infect Dis 1999;28:520-526.

10. Hellstein JW, Adler RA, Edwards B, et al; American Dental Association Council on Scientific Affairs Expert Panel on Antiresorptive Agents. Managing the care of patients receiving antiresorptive therapy for prevention and treatment of osteoporosis: Executive summary of recommendations from the American Dental Association Council on Scientific Affairs. J Am Dent Assoc 2011;142:1243-1251.

11. Wang HL, Weber D, McCauley LK. Effect of long-term oral bisphosphonates on implant wound healing: Literature review and a case report. $J$ Periodontol 2007;78: 584-594.

12. Lane N, Armitage GC, Loomer P, et al. Bisphosphonate therapy improves the outcome of conventional periodontal treatment: Results of a 12-month, randomized, placebo-controlled study. J Periodontol 2005;76: 1113-1122.

13. Sharma A, Pradeep AR. Clinical efficacy of $1 \%$ alendronate gel in adjunct to mechanotherapy in the treatment of aggressive periodontitis: A randomized controlled clinical trial. J Periodontol 2012;83:19-26.
14. Ammann P, Rizzoli R, Müller K, Slosman D, Bonjour JP. IGF-I and pamidronate increase bone mineral density in ovariectomized adult rats. Am J Physiol 1993;265: E770-E776.

15. Murakami H, Nakamura T, Tsurukami H, Abe $M$, Barbier A, Suzuki K. Effects of tiludronate on bone mass, structure, and turnover at the epiphyseal, primary, and secondary spongiosa in the proximal tibia of growing rats after sciatic neurectomy. JBone Miner Res 1994;9:1355-1364.

16. Rogers MJ, Crockett JC, Coxon FP, Mönkkönen J. Biochemical and molecular mechanisms of action of bisphosphonates. Bone 2011;49:34-41.

17. Tokuda H, Kozawa O, Harada A, Uematsu T. Tiludronate inhibits interleukin-6 synthesis in osteoblasts: Inhibition of phospholipase D activation in MC3T3-E1 cells. J Cell Biochem 1998;69:252-259.

18. Mönkkönen J, Similä J, Rogers MJ. Effects of tiludronate and ibandronate on the secretion of proinflammatory cytokines and nitric oxide from macrophages in vitro. Life Sci 1998;62:PL95-PL102.

19. Nakaya H, Osawa G, Iwasaki N, Cochran DL, Kamoi K, Oates TW. Effects of bisphosphonate on matrix metalloproteinase enzymes in human periodontal ligament cells. J Periodontol 2000;71:1158-1166.

20. Ruggiero SL, Dodson TB, Assael LA, Landesberg R, Marx RE, Mehrotra B; American Association of Oral and Maxillofacial Surgeons. American Association of Oral and Maxillofacial Surgeons position paper on bisphosphonate-related osteonecrosis of the jaws 2009 update. J Oral Maxillofac Surg 2009;67(Suppl. 5):2-12.

21. Diel IJ, Fogelman I, Al-Nawas B, et al. Pathophysiology, risk factors and management of bisphosphonateassociated osteonecrosis of the jaw: Is there a diverse relationship of amino- and non-aminobisphosphonates? Crit Rev Oncol Hematol 2007;64:198-207.

22. Adami S, Zamberlan N. Adverse effects of bisphosphonates. A comparative review. Drug Saf 1996;14:158170.

23. Reid IR. Osteonecrosis of the jaw: Who gets it, and why? Bone 2009;44:4-10.

24. Oizumi T, Yamaguchi K, Funayama H, et al. Necrotic actions of nitrogen-containing bisphosphonates and their inhibition by clodronate, a non-nitrogen-containing bisphosphonate in mice: Potential for utilization of clodronate as a combination drug with a nitrogencontaining bisphosphonate. Basic Clin Pharmacol Toxicol 2009;104:384-392.

25. Geusens P, Nijs J, Van der Perre G, et al. Longitudinal effect of tiludronate on bone mineral density, resonant frequency, and strength in monkeys. J Bone Miner Res 1992;7:599-609.

26. Bonjour JP, Ammann P, Barbier A, Caverzasio J, Rizzoli R. Tiludronate: Bone pharmacology and safety. Bone 1995;17(Suppl. 5):473S-477S.

27. Mitsuta T, Horiuchi H, Shinoda H. Effects of topical administration of clodronate on alveolar bone resorption in rats with experimental periodontitis. J Periodontol 2002;73:479-486.

28. Reddy GT, Kumar TM, Veena KM. Formulation and evaluation of alendronate sodium gel for the treatment of bone resorptive lesions in periodontitis. Drug Deliv 2005; 12:217-222.

29. Goya JA, Paez HA, Mandalunis PM. Effect of topical administration of monosodium olpadronate on experimental periodontitis in rats. J Periodontol 2006;77:1-6. 
30. Abtahi J, Agholme F, Sandberg O, Aspenberg P. Effect of local vs. systemic bisphosphonate delivery on dental implant fixation in a model of osteonecrosis of the jaw. $J$ Dent Res 2013;92:279-283.

31. Achong R, Nishimura I, Ramachandran H, Howell TH, Fiorellini JP, Karimbux NY. Membrane type (MT) 1matrix metalloproteinase (MMP) and MMP-2 expression in ligature-induced periodontitis in the rat. J Periodontol 2003;74:494-500.

32. Graves DT, Fine D, Teng YT, Van Dyke TE, Hajishengallis G. The use of rodent models to investigate host-bacteria interactions related to periodontal diseases. J Clin Periodontol 2008;35:89-105.

33. Murakami H, Takahashi N, Sasaki T, et al. A possible mechanism of the specific action of bisphosphonates on osteoclasts: Tiludronate preferentially affects polarized osteoclasts having ruffled borders. Bone 1995;17: 137-144.

34. Reszka AA, Halasy-Nagy JM, Masarachia PJ, Rodan GA. Bisphosphonates act directly on the osteoclast to induce caspase cleavage of mst1 kinase during apoptosis. A link between inhibition of the mevalonate pathway and regulation of an apoptosis-promoting kinase. J Biol Chem 1999;274:34967-34973.

35. Adami S, Bhalla AK, Dorizzi R, et al. The acute-phase response after bisphosphonate administration. Calcif Tissue Int 1987;41:326-331.

36. Weinreb M, Quartuccio H, Seedor JG, et al. Histomorphometrical analysis of the effects of the bisphosphonate alendronate on bone loss caused by experimental periodontitis in monkeys. J Periodontal Res 1994;29: 35-40.

37. Yamaguchi K, Motegi K, Iwakura Y, Endo Y. Involvement of interleukin-1 in the inflammatory actions of aminobisphosphonates in mice. Br J Pharmacol 2000; 130:1646-1654.

38. Brooks JK, Gilson AJ, Sindler AJ, Ashman SG, Schwartz KG, Nikitakis NG. Osteonecrosis of the jaws associated with use of risedronate: Report of 2 new cases. Oral Surg Oral Med Oral Pathol Oral Radiol Endod 2007;103:780-786.

39. Hokugo A, Christensen R, Chung EM, et al. Increased prevalence of bisphosphonate-related osteonecrosis of the jaw with vitamin D deficiency in rats. J Bone Miner Res 2010;25:1337-1349.

40. Davi H, Tronquet C, Caix J, et al. Disposition of tiludronate (Skelid) in animals. Xenobiotica 1999;29: 1017-1031.

41. Ishimi Y, Miyaura C, Jin CH, et al. IL- 6 is produced by osteoblasts and induces bone resorption. J Immunol 1990;145:3297-3303.
42. Roodman GD. Interleukin-6: An osteotropic factor? J Bone Miner Res 1992;7:475-478.

43. Delguste C, Amory H, Doucet $M$, et al. Pharmacological effects of tiludronate in horses after long-term immobilization. Bone 2007;41:414-421.

44. Delmas PD, Vergnaud $P$, Arlot ME, Pastoureau $P$ Meunier PJ, Nilssen MH. The anabolic effect of human PTH (1-34) on bone formation is blunted when bone resorption is inhibited by the bisphosphonate tiludronate - Is activated resorption a prerequisite for the in vivo effect of PTH on formation in a remodeling system? Bone 1995; 16:603-610.

45. Migliorati CA. Bisphosphanates and oral cavity avascular bone necrosis. J Clin Oncol 2003;21:4253-4254.

46. Brunsvold MA, Chaves ES, Kornman KS, Aufdemorte TB, Wood R. Effects of a bisphosphonate on experimental periodontitis in monkeys. J Periodontol 1992; 63:825-830.

47. Alencar VB, Bezerra MM, Lima V, et al. Disodium chlodronate prevents bone resorption in experimental periodontitis in rats. J Periodontol 2002;73:251-256.

48. Duesterdieck-Zellmer KF, Driscoll N, Ott JF. Concentrationdependent effects of tiludronate on equine articular cartilage explants incubated with and without interleukin-1ß. Am J Vet Res 2012;73:1530-1539.

49. Barou O, Lafage-Proust MH, Martel C, et al. Bisphosphonate effects in rat unloaded hindlimb bone loss model: Three-dimensional microcomputed tomographic, histomorphometric, and densitometric analyses. J Pharmacol Exp Ther 1999;291:321-328.

50. Cetinkaya BO, Keles GC, Ayas B, Gurgor P. Effects of risedronate on alveolar bone loss and angiogenesis: $A$ stereologic study in rats. J Periodontol 2008;79:19501961.

51. Fleisch H. Bisphosphonates. Pharmacology and use in the treatment of tumour-induced hypercalcaemic and metastatic bone disease. Drugs 1991;42:919-944.

52. Adachi $H$, Igarashi K, Mitani H, Shinoda H. Effects of topical administration of a bisphosphonate (risedronate) on orthodontic tooth movements in rats. J Dent Res 1994;73:1478-1486.

Correspondence: Dr. Flávia Aparecida Chaves Furlaneto, Division of Periodontics, Department of Surgery and Integrated Clinic, Dental School of Araçatuba, UNESP, São Paulo State University, Rua José Bonifácio, 1193 16015-900, Vila Mendonça, Araçatuba, SP, Brazil. Fax: 55-3636-3332; e-mail: flafurlaneto@hotmail.com.

Submitted September 25, 2013; accepted for publication December 31, 2013. 